Research Paper

\title{
Rapid Breast Cancer Disease Progression Following Cyclin Dependent Kinase 4 and 6 Inhibitor Discontinuation
}

\author{
Sami I. Bashour1, Iman Doostan², Khandan Keyomarsi², Vicente Valero', Naoto T. Ueno', Powel H. \\ Brown $^{1,3}$, Jennifer K. Litton ${ }^{1}$, Kimberly B. Koenig ${ }^{1}$, Meghan Karuturi ${ }^{1}$, Sausan Abouharb1, Debasish \\ Tripathy ${ }^{1}$, Stacy L. Moulder-Thompson ${ }^{1 *}$, Nuhad K. Ibrahim ${ }^{1 * \llbracket}$ \\ 1. Department of Breast Medical Oncology, The University of Texas MD Anderson Cancer Center, 1155 Pressler Street, Houston, TX 77030. \\ 2. Department of Experimental Radiation Oncology, The University of Texas MD Anderson Cancer Center, 1515 Holcombe Blvd, Houston, TX 77030. \\ 3. Department of Clinical Cancer Prevention, The University of Texas MD Anderson Cancer Center, 1515 Holcombe Blvd, Houston, TX 77030. \\ *Both Co-authors contributed equally to this work
}

$\square$ Corresponding author: Nuhad K. Ibrahim, MD, FACP, Professor, Department of Breast Medical Oncology, The University of Texas MD Anderson Cancer Center, 1155 Pressler Street, CPB5.3540, Houston, TX 77030-4009. Phone: +713-792-2817; Fax: +713-794-4385; Email: nibrahim@mdanderson.org

(c) Ivyspring International Publisher. This is an open access article distributed under the terms of the Creative Commons Attribution (CC BY-NC) license (https:// creativecommons.org/licenses/by-nc/4.0/). See http://ivyspring.com/terms for full terms and conditions.

Received: 2016.11.03; Accepted: 2017.05.02; Published: 2017.07.05

\begin{abstract}
Background: CDK 4 and 6 inhibitors (CDK4/6i), which arrest unregulated cancer cell proliferation, show clinical efficacy in breast cancer. Unexpectedly, a patient treated on a CDK4/6i-based trial, as first-line therapy in metastatic breast cancer, developed rapid disease progression following discontinuation of study drug while receiving standard second-line therapy off trial. We thus sought to expand this observation within a population of patients treated similarly at The University of Texas MD Anderson Cancer Center.

Methods: Using an IRB-approved protocol, 4 patients previously enrolled on CDK4/6i trials were analyzed for outcomes after discontinuing study drug. These patients were treated on a randomized trial of first-line endocrine therapy +/- a CDK4/6i. Rapid disease progression was defined as progression occurring within 4 months of CDK4/6i discontinuation.

Results: In total, 4 patients developed rapid disease progression and died; 2 of whom died within 6 months of CDK4/6i discontinuation.

Conclusion: This case series suggests a potential for rapid disease progression following CDK 4/6i discontinuation. However, the clinical course following progression must be validated in large CDK4/6i clinical trials and standard-of-care cohorts. If confirmed, such observations may alter the algorithm for subsequent therapy in patients with disease progression on CDK4/6i. Nevertheless, the need remains to define a mechanistic basis for this rapid progression and formulate alternative therapeutic strategies.
\end{abstract}

Key words: Breast Cancer; Estrogen Receptor-Positive Breast Cancer; Rapid Disease Progression; CDK and CDK Inhibitors

\section{Introduction}

In normal cellular division, various cyclin dependent kinases (CDKs) control the transition from one phase of the cell cycle to another by binding to cyclin protein subunits and overcoming specific molecular checkpoints. CDK4 and CDK6 (CDK4/6), in complex with the $\mathrm{D}$ class of cyclins, regulate the transition from the early $G_{1}$ to late $G_{1}$ phase, while cyclin $\mathrm{E}$, in complex with CDK2, regulate the transition from the late $G_{1}$ phase to the $S$ phase. The key substrate for these two different complexes is the 
retinoblastoma $(\mathrm{Rb})$ protein, which is sequentially phosphorylated in early and late $G_{1}$ by cyclin $\mathrm{D}_{1} / \mathrm{CDK} 4 / 6$, followed by cyclin E/CDK2 complexes. The resulting hyperphosphorylated $\mathrm{Rb}$ is deactivated, allowing the dividing cell to bypass the $G_{1}-S$ phase cell cycle checkpoint.

Overexpression of the $G_{1}$ cyclins accelerate the transition through the $\mathrm{G}_{1}-\mathrm{S}$ phase checkpoint, and the subsequent cellular proliferation impacts disease prognosis $[1,2,3,4]$. CDK inhibitors arrest cellular proliferation in response to various conditions. Activating aberrations, such as gene amplification and translocation of cyclins, as well as loss of function of $\mathrm{Rb}$ and $\mathrm{CDK}$ inhibitors, are common in cancer, making CDKs logical cancer therapy targets [5]. Recently, selective CDK4/6 inhibitors (CDK4/6i) have been shown to block the growth of estrogen receptor-positive (ER+) breast cancer cell lines [6], but have low activity as single agents for the treatment of breast cancer [7]. Combining CDK4/6 inhibition (palbociclib) with endocrine therapy (letrozole) significantly improved progression-free survival (PFS) from 10.2 to 20.2 months $(p=0.0004)$ when compared to letrozole alone as first-line therapy for the treatment of patients with advanced ER+, HER2-negative (ER+/HER2-) disease. This treatment combination had an acceptable toxicity profile, which included reversible neutropenia [8]. Although the study was not powered to assess overall survival (OS), the near doubling in PFS did not translate into significant improvement in OS, with a median of 37.5 months for the letrozole plus palbociclib arm, and 33.3 months in the letrozole alone arm $(p=0.42)$.

At our institution, a patient with bone-only disease, who developed cyclic neutropenia, and also had prolonged disease stability for 12 months while treated on a randomized, double-blind trial of first-line hormone therapy +/- a CDK4/6i, was noted to have increased uptake on bone scans with a rising CA15-3, prompting study drug discontinuation and the initiation of second-line therapy with everolimus and exemestane. After 2 months of second-line therapy, the patient exhibited atypical, rapid disease progression which also included visceral organ involvement. Given this patient's history of a well-controlled disease status while on study but rapid, secondary disease progression after only 2 months of second-line therapy, we further explored the rate of rapid, secondary disease progression in other patients treated at our cancer center following CDK4/6i trial discontinuation.

\section{Methods}

Using an IRB-approved protocol at the University of Texas MD Anderson Cancer Center, cases of patients who were removed from trials of hormone therapy +/- a CDK4/6i were identified and evaluated for patient outcomes during subsequent treatment after study discontinuation. Only patients who discontinued CDK4/6i therapy due to disease progression are included in this analysis. The data collected included the number of therapies received prior to entering the $\mathrm{CDK} 4 / 6 \mathrm{i}$ clinical trial, subsequent treatment regimen after discontinuing CDK4/6i, start and stop dates for subsequent systemic therapy, reason for discontinuation, and date of last follow-up or patient death. One CDK4/6i-based clinical trial was identified with enrollment durations long enough to have patients who developed disease progression. This trial was a double-blind, first-line therapy trial of CDK4/6i versus placebo in combination with an aromatase inhibitor (AI) for the treatment of advanced ER+/HER2- breast cancer. Patients enrolled in this placebo-controlled trial were allowed, per protocol, to be unblinded if requested by the treating physician at the time of disease progression. All patients had blood counts followed routinely as per protocol. For the purposes of this analysis, those who were unblinded and found to be receiving CDK4/6i, as well as those who remained blinded but developed neutropenia while receiving protocol therapy, were considered to have received the CDK4/6i, as AI therapy alone is unlikely to result in Grade III neutropenia, defined as an absolute neutrophil count (ANC) below 1,000/uL $[7,9,10]$.

Since time to treatment progression (TTP) approaches nine months in postmenopausal women receiving letrozole as first-line AI therapy [11], we defined rapid disease progression as progression that occurred within four months of CDK4/6i plus first-line AI discontinuation. Median duration of therapy on CDK4/6i trial was defined as the time from CDK4/6i trial initiation until trial discontinuation. TTP was defined as the time from CDK4/6i trial discontinuation until disease progression while receiving post $\mathrm{CDK} 4 / 6 \mathrm{i}$ trial therapy. Post CDK4/6i survival was defined as the time from CDK4/6i trial discontinuation until the time of death, and OS as the time from CDK4/6i trial initiation until the time of death (Figure 1).

\section{Results}

At the time of this report, 15 patients had been treated on the first-line, randomized placebo-controlled trial, and 6 patients had discontinued therapy due to disease progression. None of the patients were removed from the trial for any reason other than disease progression. Three of these 6 patients were unblinded; the first had 
neutropenia during therapy and found to have received the CDK4/6i, while the other two did not develop neutropenia and were found to be on the placebo arm of the trial. The remaining 3 patients, who were kept blinded following study discontinuation, developed neutropenia on trial and are presumed to have received the CDK4/6i for the purposes of this analysis (Table 1).

Table 1: Patients Who Received First-line Hormone Therapy With a CDK4/6 Inhibitor

\begin{tabular}{|c|c|c|c|c|c|c|c|c|}
\hline Pt. & Age & $\begin{array}{l}\text { Low } \\
\text { ANC }\end{array}$ & $\begin{array}{l}\text { Cycles of } \\
\text { CDK } 4 / 6 \mathrm{i} \\
\text { or } \\
\text { Placebo }\end{array}$ & $\begin{array}{l}\text { Duration } \\
\text { of } \\
\text { Therapy } \\
\text { (months) }\end{array}$ & $\begin{array}{l}\text { Post } \\
\text { CDK4/6i } \\
\text { Clinical Trial } \\
\text { Therapy }\end{array}$ & $\begin{array}{l}\mathrm{TTP}^{1} \\
\text { (months) }\end{array}$ & $\begin{array}{l}\text { Post } \\
\text { CDK4/6i } \\
\text { Survival } \\
\text { (months) }\end{array}$ & $\begin{array}{l}\text { OS } \\
\text { (months) }\end{array}$ \\
\hline 1 & 42 & Yes & 12 & 11.70 & Capecitabine & 2.80 & 24.83 & 36.53 \\
\hline 2 & 49 & Yes & 12 & 11.60 & $\begin{array}{l}\text { Exemestane } \\
+ \text { Everolimus }\end{array}$ & 2.10 & 5.20 & 16.80 \\
\hline 3 & 38 & Yes & 18 & 17.00 & $\begin{array}{l}\text { Exemestane } \\
+ \text { Everolimus }\end{array}$ & 1.46 & 10.73 & 27.73 \\
\hline 4 & 50 & Yes & 9 & 8.40 & $\begin{array}{l}\text { Exemestane } \\
+ \text { Everolimus }\end{array}$ & 2.60 & 3.70 & 12.10 \\
\hline & dian & & 12 & 11.65 & $\mathrm{~N} / \mathrm{A}$ & 2.35 & 7.97 & 22.32 \\
\hline
\end{tabular}

Legend/Abbreviations: Pt.: Patient, $\mathrm{TTP}^{1}$ : Time to progression while receiving post CDK4/6i clinical trial therapy.

The median duration of therapy on trial was 11.65 months. However, following CDK4/6i discontinuation, all 4 patients known or presumed to have received first-line AI therapy in combination with a CDK4/6i developed rapid disease progression and two patients died within 6 months of discontinuing the CDK4/6i (Table 1). Three of the 4 patients were treated with exemestane and everolimus after CDK4/6i plus first-line AI discontinuation, while one patient received capecitabine. In spite of the fact that all patients developed rapid disease progression following CDK4/6i discontinuation, post CDK4/6i survival durations were varied, with Patients \#2 and \#4, who

\begin{tabular}{|l|l|l|} 
Time on CDK4/6i Trial & Post CDK4/6i Therapy & \\
\hline & &
\end{tabular}

$\underbrace{\begin{array}{c}\text { CDK4/6i Trial } \\ \text { Discontinuation due } \\ \text { Initiation }\end{array}}_{\text {Duration of Therapy }} \begin{gathered}\begin{array}{c}\text { Post CDK4/6i Therapy } \\ \text { Discontinuation due to } \\ \text { Progression }\end{array} \\ \text { OS Post CDK4/6i Survival }\end{gathered}$

Figure 1: Patient Timeline of CDK4/6i Trial Participation and Outcomes Definition. Legend: Duration of Therapy is defined as the time from CDK4/6i trial initiation until trial discontinuation. TTP: Time to treatment progression; defined as the time from CDK4/6i trial discontinuation until disease progression while receiving post CDK4/6i trial therapy. Post CDK4/6i survival is defined as the time from CDK4/6i trial discontinuation until time of death. OS: Overall Survival; defined as the time from CDK4/6i trial initiation until time of death. progressed rapidly on exemestane and everolimus, expiring in 5.20 and 3.70 months, respectively, while Patients \#1 and \#3, who rapidly progressed on capecitabine and exemestane and everolimus, respectively, proceeded to survive on subsequent lines of therapy for 24.83 and 10.73 months, respectively.

\section{Discussion}

In early studies for the treatment of advanced ER+/HER2- breast cancer, CDK4/6i combined with AI showed very promising activity. However, after we observed one patient develop rapid, secondary disease progression in 63 days while receiving second-line everolimus and exemestane therapy following CDK4/6i trial discontinuation, we conducted a review of 4 patients to further characterize rapid disease progression after CDK4/6i cessation.

As previously mentioned, Finn et al. (2015) [8] reported a significantly improved PFS from 10.2 to 20.2 months after combining CDK4/6 inhibition (palbociclib) with hormone therapy (letrozole) when compared to letrozole alone as first-line therapy for the treatment of patients with advanced ER+, HER2-negative (ER+/HER2-) disease. However, although our patients received a CDK4/6i plus first-line hormone therapy, our reported cases did not show similar clinical benefit (median duration on trial of 11.65 months). Instead, these patients showed a clinical picture more consistent with single AI agent administration [11]. Nevertheless, when the CDK4/6i was discontinued, all 4 patients reflected this unanticipated, rapid, secondary disease progression on subsequent lines of therapy, which is the subject of this case series. Despite rapid disease progression, however, patients did not demonstrate consistent sensitivity or responsiveness to subsequent lines of therapy. Two of the three patients who rapidly progressed on exemestane and everolimus survived less than 6 months following CDK4/6i discontinuation, while the one patient who rapidly progressed on capecitabine and the third patient who rapidly progressed on exemestane and everolimus, survived for greater than 24 and 10 months, respectively. Of note, one patient (data not shown) had been enrolled in a separate, open-label, single-arm trial of second or greater-line endocrine therapy plus a CDK4/6i and the 
mTOR inhibitor everolimus. After 8.5 months on this CDK4/6i-based trial, the patient progressed and was treated with MLN0128, an mTORC1/2 inhibitor, plus ziv-Aflibercept, a VEGF inhibitor. On this initial post CDK4/6i therapy, the patient developed a TTP of 4.10 months, but then proceeded to respond on subsequent lines of therapy for over 20 months, and is still alive as of last follow up. Therefore, in addition to highlighting the phenomenon of rapid disease progression after CDK4/6i discontinuation, this case series also suggests that post CDK4/6i therapy may result in aberrant signaling pathways which confer primary resistance to certain therapies but not others, as occurred with Patients \#2 and \#4 and Patients \#1 and \#3, respectively, as well as with the patient who received MLN0128 plus ziv-Aflibercept following CDK4/6i discontinuation.

Additionally, patients who received exemestane as a second or greater-line therapy, in combination with everolimus in the pivotal BOLERO-2 trial, were shown to progress after 6.9 months of therapy [12], while our patient cohort who received exemestane, as second or greater-line therapy, plus everolimus following CDK4/6i discontinuation, had a median TTP of 2.1 months. Moreover, while our report focuses on a select group of patients with rapid disease progression on subsequent anti-estrogen therapy following CDK4/6i discontinuation, a recent follow-up analysis from the PALOMA-1/TRIO-18 trial by Finn et al. (2016) [13] reported a similar median duration of subsequent endocrine therapy in both study arms (palbociclib plus letrozole versus letrozole alone), but does not address the issue of rapid disease progression as we did. Similarly, Turner et al. (2016) [14] investigated post treatment outcomes in patients who received palbociclib plus fulvestrant versus fulvestrant alone in the PALOMA-3 trial. In their analysis, the median duration on next-line therapy was 17.9 months in the palbociclib plus fulvestrant cohort [14], however they did not assess time to progression on next-line therapy according to therapy used. As our case series suggests that CDK4/6i therapy may confer primary resistance to specific next-line therapies, further analysis into post CDK 4/6i outcomes according to subsequent therapy used is warranted.

After accounting for the effects of hormone therapy and everolimus, a greatly reduced TTP raises the issue of a specific CDK4/6i mechanism of resistance and/or the activation of a yet to be defined biological pathway that results from CDK4/6i withdrawal. In a recent study by Vijayaraghavan et al. (2017) [15], resistance to palbociclib was found to be related to the presence of retinoblastoma $(\mathrm{Rb})$ and low molecular weight cyclin E (LMW-E), where higher levels of $\mathrm{Rb}$ and LMW-E in breast cancer cell lines greatly reduced sensitivity to palbociclib. Thus, although the underlying mechanisms of these clinical observations remains unknown, preclinical data may offer potential explanations not only for the rapid disease progression seen in certain patients following CDK4/6i discontinuation, but a better understanding as to which patients are less likely to respond to CDK4/6i prior to therapy initiation.

Inhibition of CDK4/6 has been associated with senescence-autophagy transition (SAT) in stromal fibroblasts leading to mitochondrial dysfunction and catabolism. This process releases high-energy mitochondrial fuels (onco-catabolites) into the stroma, such as L-lactate, ketone bodies, and glutamine, which act to drive anabolic tumor growth and cancer metastasis [16, 17]. Indeed, when fibroblasts with induced senescence were co-cultured with MDA-MB-231 breast cancer cell lines, a near 2-fold increase in tumor growth, without increased angiogenesis, was noted as compared to control [16]. Thus, rapid progression following CDK4/6i discontinuation may be explained by the fact that although these onco-catabolites are generated in patients receiving $\mathrm{CDK} 4 / 6 \mathrm{i}$, their tumor-growing effects are silenced by the significant anti-proliferative properties of CDK4/6i during active therapy. When CDK4/6i are discontinued, however, the suppression of these onco-catabolites is released, potentially leading to rapid tumor progression.

This hypothesis-generating report will need further investigation in larger patient cohorts, either by collecting subsequent therapy outcome data in existing CDK4/6i clinical trials, or by determining patient outcomes after the FDA-accelerated approval of palbociclib made the drug available to patients with advanced ER+/HER2- breast cancer. Of importance, patients in this series received an AI with mTOR inhibition following the discontinuation of a CDK4/6i clinical trial. Preclinical experiments have suggested that inhibition of cyclin $\mathrm{D}_{1}$, or its other binding partners to CDK4/6, enhance features of the epithelial-mesenchymal transition (EMT) and increase mobility in both breast and pancreatic cancer cell lines, which occurs predominantly through the activation of TGF- $\beta$, inducing Akt-TOR signaling [18, 19]. Thus, while one may expect everolimus to play a role in overcoming this potential pathway of CDK4/6i resistance, its effects may not be observed following CDK4/6i discontinuation due to the presence of onco-catabolites and the establishment of a primed microenvironment that is highly conducive to rapid tumor growth. As such, continuation of CDK4/6 inhibition may be necessary for maximal effect when considering a targeted therapy as subsequent 
treatment after CDK4/6i. Additionally, biologic studies to identify patients at risk for developing rapid disease progression should also be pursued as these patients may need more aggressive monitoring for progression or require chemotherapy as subsequent therapy after CDK4/6i.

\section{Conclusion}

The markedly shortened TTP observed in our set of patients who discontinued CDK4/6i raises concerns as to the effect of CDK4/6i on metastatic breast cancer disease biology. In addition to the observed phenomenon of rapid disease progression, response to subsequent therapies was found to be varied in patients, with two of the patients developing rapid disease progression after CDK4/6i withdrawal, but then proceeding to follow the expected natural history of disease (surviving 10.73 and 24.83 months after CDK4/6i discontinuation), and two patients developing rapid progression and then expiring in 5.20 and 3.70 months after CDK4/6i discontinuation. As explanations for these unexpected disease outcomes remain largely unexplored, and the mechanisms leading to rapid disease progression have yet to be elucidated, such findings warrant further investigation using larger datasets and prospective outcome studies.

\section{Abbreviations}

CDK: Cyclin Dependent Kinase; CDK 4/6i: Cyclin Dependent Kinase 4 and 6 inhibitors; ER+/HER2-: Estrogen Receptor Positive/Human Epidermal Growth Factor Receptor 2 Negative; PFS: Progression Free Survival; OS: Overall Survival; TTP: Time To Progression; IRB: Institutional Review Board; AI: Aromatase Inhibitor; mTOR: Mammalian Target of Rapamycin; ANC: Absolute Neutrophil Count; LMW-E: Low Molecular Weight Cyclin E; Rb: Retinoblastoma; SAT: Senescence-Autophagy Transition; EMT: Epithelial-Mesenchymal Transition.

\section{Acknowledgement}

This work was supported by the Nellie B. Connally Breast Center at the University of Texas MD Anderson Cancer Center.

\section{Ethics Approval and Consent to Participate}

All appropriate guidelines and legislation in conducting the study were followed. The University of Texas MD Anderson Cancer Center Institutional Review Board approved this study. The research has not been and will not be submitted simultaneously to another journal, in whole or in part.

\section{Consent for Publication}

All necessary consent was obtained prior to research and publication.

\section{Availability of Data and Material}

All data and materials are presented in the manuscript.

\section{Authors' Contributions}

All named authors have contributed to the writing of this paper and designing of the study. They have read the manuscript and have agreed to submit the paper to the Journal of Cancer in its present form.

\section{Competing Interests}

All authors, Sami I. Bashour, Iman Doostan, Khandan Keyomarsi, Vicente Valero, Naoto T. Ueno, Powel H. Brown, Jennifer K. Litton, Kimberly B. Koenig, Meghan Karuturi, Sausan Abouharb, Debasish Tripathy, Stacy L. Moulder-Thompson, and Nuhad K. Ibrahim, have no conflicts of interest or competing interests to declare.

\section{References}

1. Resnitzky D, Gossen M, Bujard H, Reed SI. Acceleration of the G1/S Phase Transition by Expression of Cyclins D1 and E with an Inducible System. Mol Cell Biol. 1994;14(3):1669-1679.

2. An HX, Beckmann MW, Reifenberger G, Bender HG, Niederacher D. Gene Amplification and Overexpression of CDK4 in Sporadic Breast Carcinomas Is Associated with High Tumor Cell Proliferation. Am J Pathol. 1999;154(1):113-118.

3. Keyomarsi K, Tucker SL, Buchholz TA, Callister M, Ding Y, Hortobagyi GN, Bedrosian I, Knickerbocker C, Toyofuku W, Lowe M, Herliczek TW, Bacus SS. Cyclin E and Survival in Patients with Breast Cancer. N Engl J Med. 2002;347(20):1566-1575.

4. Arnold A, Papanikolaou A. Cyclin D1 in Breast Cancer Pathogenesis. J Clin Oncol. 2005;23(18):4215-4224.

5. VanArsdale T, Boshoff C, Arndt KT, Abraham RT. Molecular Pathways: Targeting the Cyclin D-CDK4/6 Axis for Cancer Treatment. Clin Cancer Res. 2015;21(13):2905-2910.

6. Finn RS, Dering J, Conklin D, Kalous O, Cohen DI, Desai AJ, Ginther C, Atefi M, Checn I, Fowst C, Los G, Slamon DJ. PD 0332991, A Selective Cyclin D Kinase 4/6 Inhibitor, Preferentially Inhibits Proliferation of Luminal Estrogen Receptor-Positive Human Breast Cancer Cell Lines In Vitro. Breast Cancer Res. 2009;11(5):R77. DOI: $10.1186 /$ bcr2419.

7. DeMichele A, Clark AS, Tan KS, Heitjan DF, Gramlich K, Gallagher M, Lal P, Feldman M, Zhang P, Colameco C, Lewis D, Langer M, Goodman N, Domchek S, Gogineni K, Rosen M, Fox K, O'Dwyer P. CDK4/6 Inhibitor Palbociclib (PD0332991) in Rb+ Advanced Breast Cancer: Phase II Activity, Safety, and Predictive Biomarker Assessment. Clin Cancer Res. 2015;21(5):995-1001.

8. Finn RS, Crown JP, Lang I, Boer K, Bondarenko IM, Kulyk SO, Ettl J, Patel R, Pinter T, Schmidt M, Shparyk Y, Thummala AR, Voytko NL, Fowst C, Huang X, Kim ST, Randolph S, Slamon DJ. The Cyclin-Dependent Kinase 4/6 Inhibitor Palbociclib in Combination with Letrozole Versus Letrozole Alone as First-Line Treatment of Estrogen Receptor-Positive, HER2-Negative, Advanced Breast Cancer (PALOMA-1/TRIO-18): A Randomized Phase 2 Study. Lancet Oncol. 2015;16(1):25-35.

9. Turner NC, Ro J, Andre F, Loi S, Verma S, Iwata H, Harbeck N, Loibl S, Bartlett CH, Zhang K, Giorgetti C, Randolph S, Koehler M, Cristofanilli M. Palbociclib in Hormone-Receptor-Positive Advanced Breast Cancer. N Engl J Med. 2015;373(3):209-219.

10. Flowers CR, Seidenfeld J, Bow EJ, Karten C, Gleason C, Hawley DK, Kuderer NM, Langston AA, Marr KA, Rolston KVI, Ramsey SD. Antimicrobial Prophylaxis and Outpatient Management of Fever and Neutropenia in Adults Treated for Malignancy: American Society of Clinical Oncology Clinical Practice Guideline. J Clin Oncol. 2013;31(6):794-810.

11. Mouridsen $H$, Gershanovich $M$, Sun $Y$, Perez-Carrion R, Boni C, Monnier A, Apffelstaedt J, Smith R, Sleeboom HP, Jaenicke F, Pluzanska A, Dank M, Becquart D, Bapsy PP, Salminen E, Snyder R, Chaudri-Ross H, Lang R, Wyld P, Bhatnagar A. Phase III Study of Letrozole Versus Tamoxifen as First-Line Therapy of Advanced Breast Cancer in Postmenopausal Women: Analysis of 
Survival and Update of Efficacy From the International Letrozole Breast Cancer Group. J Clin Oncol. 2003;21(11):2101-2109.

12. Baselga J, Campone M, Piccart M, Burris HA III, Rugo HS, Shamoud T, Shinzaburo N, Gnant M, Pritchard KI, Lebrun F, Beck JT, Ito Y, Yardley D, Deleu I, Perez A, Bachelot T, Vittori L, Xu Z, Mukhopadhyay P, Lebwohl D, Hortobagyi GN. Everolimus in Postmenopausal Hormone-Receptor-Positive Advanced Breast Cancer. N Engl J Med. 2012;366(6):520-529.

13. Finn RS, Crown JP, Ettl J, Pinter T, Thummala A, Shparyk Y, Patel R, Randolph S, Kim S, Huang X, Nadanaciva S, Huang Bartlett C, Slamon DJ. Treatment patterns of post-disease progression in the PALOMA-1/TRIO-18 trial. [abstract]. In: Proceedings of the Thirty-Eighth Annual CTRC-AACR San Antonio Breast Cancer Symposium: 2015 Dec 8-12; San Antonio, TX. Philadelphia (PA): AACR; Cancer Res 2016; 76(4 Suppl): Abstract nr P4-13-02.

14. Turner NC C, André $\mathrm{F}$, Cristofanilli M, Verma S, Iwata H, Loi S, Harbeck N, Ro J, Colleoni M, Zhang K, Huang Bartlett C, Giorgetti C, Slamon D. Treatment postprogression in women with endocrine-resistant HR+/HER2advanced breast cancer who received palbociclib plus fulvestrant in PALOMA-3. [abstract]. In: San Antonio Breast Cancer Symposium: 2016 Dec 6-10; San Antonio, TX. Abstract nr P4-22-06.

15. Vijayaraghavan S, Karakas C, Doostan I, Chen X, Bui T, Yi M, Raghavendra AS, Zhao Y, Bashour SI, Ibrahim NK, Karuturi M, Wang J, Winkler JD, Amaravadi RK, Hunt KK, Tripathy D, Keyomarsi K. CDK4/6 and autophagy inhibitors synergistically induce senescence in $\mathrm{Rb}$ positive cytoplasmic cyclin E negative cancers. Nat Commun. 2017 Jun 27;8:15916. doi: $10.1038 /$ ncomms15916.

16. Capparelli C, Chiavarina B, Whitaker-Menezes D, Pestell TG, Pestell RG, Hulit J, Ando S, Howell A, Martinez-Outschoorn UE, Sotgia F, Lisanti MP. CDK Inhibitors (p16/p19/p21) Induce Senescence and Autophagy in Cancer-Associated Fibroblasts, "Fueling" Tumor Growth via Paracrine Interactions, without an increase in neo-angiogenesis. Cell Cycle. 2012;11(19):3599-3610.

17. Capparelli C, Guido C, Whitaker-Menezes D, Bonuccelli G, Balliet R, Pestell TG, Goldberg AF, Pestell RG, Howell A, Sneddon S, Birbe R, Tsirigos A, Martinez-Outschoorn U, Sotgia F, Lisanti MP. Autophagy and Senescence in Cancer-Associated Fibroblasts Metabolically Supports Tumor Growth and Metastasis Via Glycolysis and Ketone Production. Cell Cycle. 2012;11(12):2285-2302.

18. Tobin NP, Sims AH, Lundgren KL, Lehn S, Landberg G. Cyclin D1, Id1 and EMT in Breast Cancer. BMC Cancer. 2011;11:417.

19. Liu F, Korc M. CDK4/6 Inhibition Induces Epithelial-Mesenchymal Transition and Enhances Invasiveness in Pancreatic Cancer Cells. Mol Cancer Ther. 2012;11(10):2138-2148. 